\title{
A NOTE ON THE DENISOVA CAVE mtDNA SEQUENCE
}

The recent publication of a mtDNA sequence by Krause, et al.(2010) produced a proposal by the authors that the differences between this sequence, that of Modern Humans and Neandertal sequences indicated that the Denisova individual was probably derived from an unknown hominid population that shared its last common ancestor with AMH and Neandertals before 1.0 mya. The reason for this speculation was the great number of base pair differences between the Denisova sample and AMH and Neandertal samples. While there is significant evidence of degradation present in the reported sequence which parallels degraded mtDNA as Caldararo and Gabow (2000) argued in a paper in Ancient Biomolecules, and Caldararo (2004) extended in a later analysis, some sequences do align with published samples. Our analysis leads us to conclude, however, that the Denisova sample was significantly degraded and the resulting sequence up to 16193 contains corrupted mtDNA. After 16193 if one reads into the sequence a break as appears in the Krings, et al. 1997 as an insert of a cytocine between 16193 and 16194. We note this insert in the Caldararo \& Gabow 2000 paper where we align several modern reference sequences with several ancient sequences both identified from Neandertal material (Feldhofer consensus) and early moderns. There is another insert between 16262 and 16263 in the Krings et al. sequence for Feldhofer but this does not appear in the Denisova sequence. If one adds the Denisova Cave sequence to our alignment (see figure 1) and begin the reading from the Neandertal insert we find that the Denisova sequence then fits the reference sequence of Anderson and that used by Krings (1997) with only a few bp variations (18). The same is not true when one reaches 16264. There is also an insert between 16263 and 16264 in the 
Neandertal sequence but if one reads ignoring the Neandertal insert using the Denisova data the reading is nearly identical to the contemporary modern human sequence.

This changes the appearance of the Denisova sequence and makes it appear to reflect a combination of Neandertal sequences and AMH sequences consistent with the recent analysis of the Neandertal genome by Green et al. (2010). In the entire sequence from 16020 to 16409 the adjusted Denisova sequence agrees with the Neandertal sequence of Krings, et al. 1997, 17 times where both do not agree with the Anderson sequence. But the Neandertal sequence differs from the Denisova sequence but agrees with the Anderson sequence in other 124 locations but of these 113 are found up stream of the $\mathrm{C}$ insert between location 16193 and 16194. Of the other ancient mtDNA sequences we provide in our 2000 chart, Betty (1996) agrees 19 times with Denisova where variations occur from the Anderson sequence, Horai and Hayasaka (1990) 17 times, Handt (1996) 8 times and Handt (1994) 4 times. The Betty sample is from Australian Aborigines and the Horai and Hayasaka from Japan.

The recognition of the Neandertal inserts in the Denisova sequence changes the reading considerably and indicates that the sequence before the insert at 16194, perhaps ending at 16181 is corrupt either from degradation or during preparation for sequencing. There may be another explanation for the lack of sequence alignment before that location and the substantial agreement after it. Nevertheless, this finding argues for modern human status of the Denisova sample and against a new species designation as suggested from the original analysis (Krause, et al., 2010). Variations in mtDNA in populations and their significance given the natural history of mitochondria have been noted by Ballard and Whitlock (2004) with a caution of their use to build phylogenetic relationships. 
What is also interesting is that the Genbank Neandertal Feldhofer sequence (FM865407.1) differs substantially from the clones published in the original ell article by Krings, et al. (1997). In the original paper the various clones are shown aligned together with the Anderson reference sample in Figures 4 and 5 of their paper beginning in Figure 4 at 16,022 in Figure 4 and running to 16,401 in Figure 5 with the Neandertal consensus of the clones from the different laboratories at the bottom. For example an “A” appears at position 16,037 in the Anderson reference sample but a consensus of the clones for the Neandertal finds a " $G$ ” at this site. No other differences are seen in the sequence for the Feldhofer as consensus before this site except in two clones at position 16,036. In the Genbank sequence for Feldhofer we find 10 differences listed. These identical differences are found in the Genbank sequences for Neandertal isolates from Mezmaiskaya 1, Vindija 33.25, and Sidron 1351e. It is difficult to explain this situation and we will not venture an explanation but would hope that one is available from the laboratories involved.

Niccolo Caldararo, Ph.D.

\section{Bibliography}

Betty, D.J.; Chin-Atkins, A.N.; Croft, L.; Sraml, M. and Easteal, S., (1996) "Multiple independent origins of the COII / fRNAlys intergenic 9-bp mtDNA deletion in Aboriginal Australians,” Am. J. Hum. Genet. , v. 58:428-433.

Ballard, J.W. \& Whitlock, M.C., (2004) “The incomplete natural history of mitochondria,” Mol.Evol., Apr. v. 13, n. 4, pp 729-744.

Caldararo, Niccolo,(2004) " Some effects of the use of ultrasonic devices in conservation and the question of standards for cleaning objects,” Objects Specialty Group Postprints, American Institute for Conservation of Historic and Artistic Works, v.11, pp 126-153.

Caldararo, Niccolo \& Gabow, Steve, (2000) "Mitochondrial DNA analysis and the place of Neandertals in Homo,” Ancient Biomolecules, v. 3: 135-158. 
Green, Richard E.; Krause, Johannes; Briggs, Adrian W., et al., (2010) “A draft sequence of the Neandertal genome,” Science, v. 328:710-722.

Handt, O.; Krings, M.; Ward, R.H. \& Paabo, S., (1996) “The retrieval of ancient human DNA sequences,” Am.J.Hum.Genet.,v. 59, pp. 368-376.

Horai, S. \& Hayasaka, K., (1990) “Intraspecific nucleotide sequence differences in the major noncoding region of human mitochondrial DNA,” Am. J. Hum. Genet., v. 46, pp. 828-842.

Krause, Johannes; Fu, Qiaomei; Good, Jeffrey M.; Viola, Bence; Shunkov, Michael; Derevianko, Anatoli and Paabo, Svante, (2010) "The complete mitochondrial DNA genome if an unknown hominin from southern Siberia,” Nature, v. 464:894-897. 